\title{
Editorial: structural plasticity induced by drugs of abuse
}

\author{
M. Foster Olive ${ }^{1 *}$ and Justin T. Gass ${ }^{2}$ \\ ${ }^{1}$ Behavioral Neuroscience Area, Department of Psychology, Arizona State University, Tempe, AZ, USA, ${ }^{2}$ Department of \\ Neurosciences, Medical University of South Carolina, Charleston, SC, USA
}

Keywords: addiction, plasticity, glutamate, neurotrophin, GABA, homer, oligodendrocyte, cytoskeleton

The roots of addiction are often attributed to the ability of repeated drug use to compromise proper functioning of the central nervous system. Drug-induced functional changes in the brain occur on many levels, including altered expression of specific genes via genomic and epigenetic mechanisms, induction of synaptic plasticity and other cellular adaptations, and volumetric changes in discrete brain regions (Luscher and Malenka, 2011). These alterations can be drug class-specific and occur in both neuronal and non-neuronal cell populations. Drug-induced plasticity, or "rewiring" of the brain contributes to the development, maintenance, and persistence of the addicted state (Kalivas and O'Brien, 2008). The goal of this Research Topic is to assimilate recent findings related to plasticity and structural alterations produced by drug of abuse in neurons, glia, and other cell types of the brain.

The mesolimbic dopamine system, originating in the ventral tegmental area (VTA) of the midbrain and projecting rostrally to the nucleus accumbens (NAc) and prefrontal cortex (PFC), mediates the acute reinforcing and incentive salience of abused drugs, as well as their aversive properties. As reviewed by Vashchinkina et al. (2014) mesolimbic dopamine neurons are highly regulated by intrinsic and extrinsic inhibitory GABAergic neurons. Vashchinkina et al. (2014) hypothesize that abused drugs induce structural and functional mesolimbic adaptations differentially via endogenous (e.g., THIP and neurosteroids) vs. exogenous (e.g., benzodiazepines and alcohol) modulators of $\mathrm{GABA}_{\mathrm{A}}$ receptors, as well as via synaptic vs. extrasynaptic $\mathrm{GABA}_{\mathrm{A}}$ receptors in the VTA. Collo et al. (2014) review evidence that drug-induced plasticity in mesolimbic dopaminergic neurons is mediated by common dopamine and brain-derived neurotrophic factor (BDNF) signaling pathways, specifically those recruiting MEK-ERK1/2, and PI3K-Akt-mTOR. Cadet and Bisagno (2014) review evidence for substantial plasticity in non-neuronal cell types in brain reward circuitry, including changes in astrocytic glutamate transporter expression, increases in pro-inflammatory cytokine production by microglia, and dysregulated oligodendrocytic myelin production. This latter topic is further elaborated on by Somkuwar et al. (2014) in their review of the proteoglycan neuron-glial antigen 2 (NG2), its expression by oligodendrocyte progenitor cells in the brain reward circuitry, and its interaction with stress-related neuromodulators. In addition, Bajo et al. (2015) provide evidence that interleukin-1 $\beta$ alters both basal and ethanol-facilitated GABAergic transmission in the central nucleus of the amygdala, part of the extended amygdala circuitry which is implicated in ethanol's central effects.

Drugs of abuse also alter the dynamics and microstructure of both dendrites and dendritic spines, which can be interpreted as cellular (mal)adaptations that reinforce the addiction cycle (Luscher and Malenka, 2011; Gipson et al., 2014). DePoy et al. (2014) show that repeated exposure of early adolescent mice to cocaine produces lasting reductions in orbitofrontal cortex dendritic arbor length and complexity and as well as impaired reversal learning. In addition, these investigators show that while mice carrying a heterozygous deletion of the actin cytoskeleton stabilizing protein p190RhoGAP 
exhibit enhanced vulnerability to cocaine-induced hyperlocomotion, orbitofrontal dendritic complexity in cocainenaïve mice is intact. These findings suggest that orbitofrontal dendrite structure is impacted by repeated cocaine during adolescence, but pre-existing structural dendritic deficiencies do not account for increased behavioral sensitivity to this drug.

Glutamate is the predominant excitatory amino acid in the central nervous system and mediates both normal and maladaptive cellular plasticity. Several articles in this Research Topic provide novel findings on the role of glutamate in addictive processes. Weiland et al. (2015) demonstrate that antibiotics with the glutamate and GABA modulating properties (ceftriaxone and cefazolin, respectively) attenuate cue-primed reinstatement of alcohol-seeking. Griffin et al. (2015) demonstrate that chronic intermittent ethanol exposure results in increased extracellular levels of glutamate in the NAc in ethanol-dependent mice, but these effects are not a result of locally dysregulated sodiumdependent and independent glutamate transporter function, suggesting that extrinsic corticostriatal glutamatergic pathways

\section{References}

Bajo, M., Varodayan, F. P., Madamba, S. G., Roberts, A. J., Casal, L. M., Oleata, C., et al. (2015). Interleukin-1 interacts with ethanol effects on GABAergic transmission in the mouse central amygdala. Front. Pharmacol. 6:49. doi: 10.3389/fphar.2015.00049

Cadet, J. L., and Bisagno, V. (2014). Glial-neuronal ensembles: partners in drug addiction-associated synaptic plasticity. Front. Pharmacol. 5:204 doi: 10.3389/fphar.2014.00204

Collo, G., Cavalleri, L., and Spano, P. F. (2014). Structural plasticity in mesencephalic dopaminergic neurons produced by drugs of abuse: critical role of BDNF and dopamine. Front. Pharmacol. 5:259 doi: 10.3389/fphar.2014. 00259

DePoy, L. M., Perszyk, R. E., Zimmermann, K. S., Koleske, A. J., and Gourley, S. L. (2014). Adolescent cocaine exposure simplifies orbitofrontal cortical dendritic arbors. Front. Pharmacol. 5:228. doi: 10.3389/fphar.2014. 00228

Gipson, C. D., Kupchik, Y. M., and Kalivas, P. W. (2014). Rapid, transient synaptic plasticity in addiction. Neuropharmacology 76, 276-286. doi: 10.1016/j.neuropharm.2013.04.032

Griffin, W. C., Ramachandra, V. S., Knackstedt, L. A., and Becker, H. C. (2015). Repeated cycles of chronic intermittent ethanol exposure increases basal glutamate in the nucleus accumbens of mice without affecting glutamate transport. Front. Pharmacol. 6:27. doi: 10.3389/fphar.2015.00027

Kalivas, P. W., and O'Brien, C. (2008). Drug addiction as a pathology of staged neuroplasticity. Neuropsychopharmacology 33, 166-180. doi: 10.1038/sj.npp.1301564 may contribute to this hyperglutamatergic state. Finally, McGuier et al. (2015) show that deletion of the excitatory postsynaptic scaffolding protein Homer2 is associated with an increased density of long, thin dendritic spines in NAc core medium spiny neurons, yet unexpectedly these structural modifications are not modified by repeated alcohol exposure.

Together, this body of work indicates complex interactions between drugs of abuse, endogenous neuromodulators and their signaling targets, and the mechanisms underlying the functional and structural plasticity in the brain. Research into this complexity is only in its infancy, and needs to be pursued at multiple levels in order to better understand and treat addictive disorders.

\section{Acknowledgments}

The authors wish to acknowledge the funding support of NIH grants AA020537, AA013852, DA024355, DA025606, and DA037741.

Luscher, C., and Malenka, R. C. (2011). Drug-evoked synaptic plasticity in addiction: from molecular changes to circuit remodeling. Neuron 69, 650-663. doi: 10.1016/j.neuron.2011.01.017

McGuier, M. S., Padula, A. E., Mulholland, P. J., and Chandler, L. J. (2015). Homer2 deletion alters dendritic spine morphology but not alcohol-associated adaptations in GluN2B-containing NMDA receptors in the nucleus accumbens. Front. Pharmacol. 6:28. doi: 10.3389/fphar.2015.00028

Somkuwar, S. S., Staples, M. C., Galinato, M. H., Fannon, M. J., and Mandyam, C. D. (2014). Role of NG2 expressing cells in addiction: a new approach for an old problem. Front. Pharmacol. 5:279. doi: 10.3389/fphar.2014.00279

Vashchinkina, E., Panhelainen, A., Aitta-aho, T., and Korpi, E. R. (2014). GABA $A$ receptor drugs and neuronal plasticity in reward and aversion: focus on the ventral tegmental area. Front. Pharmacol. 5:256. doi: 10.3389/fphar.2014.00256

Weiland, A., Garcia, S., and Knackstedt, L. A. (2015). Cefazolin and ceftriaxone attenuate the cue-primed reinstatement of alcohol-seeking. Front. Pharmacol. 6:44. doi: 10.3389/fphar.2015.00044

Conflict of Interest Statement: The authors declare that the research was conducted in the absence of any commercial or financial relationships that could be construed as a potential conflict of interest.

Copyright (C) 2015 Olive and Gass. This is an open-access article distributed under the terms of the Creative Commons Attribution License (CC BY). The use, distribution or reproduction in other forums is permitted, provided the original author(s) or licensor are credited and that the original publication in this journal is cited, in accordance with accepted academic practice. No use, distribution or reproduction is permitted which does not comply with these terms. 